\title{
Ecología del Homo sapiens y su relación sociocultural
}

\author{
Homo sapiens ecology and his sociocultural relationship
}

Marco Barquero Arroyo ${ }^{1}$

Recibido: 27/9/2016 / Aprobado: 23/5/2017

\begin{abstract}
Resumen
Muchos individuos de la especie Homo sapiens consideran estar separada del resto de criaturas del Reino Animal. Esto implica que hay un desligamiento de las relaciones entre el ser humano y el entorno natural, cuyas causas son variadas y sus consecuencias suelen ser nocivas. El presente ensayo repasa las características ecológicas del ser humano, como por ejemplo el número de individuos, su distribución en el planeta, la esperanza de vida y las relaciones con otras especies y el entorno. Además, se analizan las repercusiones socioculturales que generan las acciones de nuestra especie sobre el entorno. Las características ecológicas del ser humano evidencian que no deberíamos considerarnos como la especie más importante del planeta, sino más bien, como integral de los ecosistemas. No obstante, los impactos producidos por el ser humano sobre los ecosistemas, tanto sobre los elementos abióticos como los bióticos, suelen ser nocivos y afectan a todas las especies, incluida la nuestra. Por lo tanto, se sugiere una reevaluación de nuestro estatus como especie y nuestra función en el ambiente.
\end{abstract}

Palabras clave: Ser humano, ecología, interacción social, impacto ambiental

Abstract

Many individuals of the specie Homo sapiens consider themselves to be separated from other creatures of the Animal Kingdom. This implies that there is a detachment of the relationship between human beings and the natural environment, whose causes are diverse and the consequences usually harmful. This essay reviews ecological human beings characteristics, as for example, number of individuals, their distribution on the planet, life expectancy and the relationship with other species and the environment. Besides, the sociocultural impacts generated from the actions of our specie in the environment. The ecological characteristics of human beings demonstrate that we should not consider ourselves as the most important specie on the planet but rather, as an integral part of the ecosystems. However, the impacts produce by human beings on ecosystems as much as on the abiotic elements as well as biotic ones are usually harmful and they affect all species, including ours. Thus, it is suggested a reevaluation of our status as a specie and our role in the environment.

Key Words: Human being, ecology, social interaction, environmental impact.

\footnotetext{
${ }^{1}$ Doctor en Ciencias Biológicas, Macquaire University, Sydney, Australia. Docente, investigador y coordinador del Bachillerato Turismo Ecológico en Sede del Caribe, Universidad de Costa Rica.

Correo electrónico: marco.barquero_a@ ucr.ac.cr / marcobarq@gmail.com
} 


\section{Introducción}

La especie humana, Homo sapiens, no se diferencia en gran medida del resto de mamíferos que habitan el planeta (Darwin, 1872). Nuestro ADN es casi idéntico al de nuestro "primo" más cercano, el chimpancé (Pan troglodytes) (Wimmer et al., 2002), ya que presentamos comportamientos similares a los observados en muchas otras especies, incluyendo algunas que no pertenecen a nuestra misma clase taxonómica (Mammalia). No obstante, una gran cantidad de personas suele considerar nuestra especie como algo separado del reino animal, la cúspide de la evolución o la creación. Esta actitud tiene importantes repercusiones sobre la forma como nos relacionamos con otras especies de nuestro entorno y cómo nos percibimos (Steffen et al., 2011). Por ejemplo, la relación humano-animal ha cambiado constantemente a través de la historia y entre sociedades humanas (Franklin y White, 2001), donde los animales han sido considerados hasta hace unos 60 años como simples máquinas sin emociones, personalidad ni cultura. Estudios recientes (por ejemplo, Whiten, 2011) indican que tales atributos no son exclusivos del ser humano y se han identificado en un número significativo de especies no humanas. La forma en la cual cada ser humano se relaciona con otras especies está ligada con el comportamiento expresado durante las relaciones humano-humano, por lo que el desligarse del entorno y el ejercer una relación de poder sobre otras criaturas afecta negativamente, no sólo, a la biodiversidad que nos rodea, sino también, las relaciones interpersonales de nuestra propia especie (Dhont et al., 2014).

En este ensayo se pretende analizar aspectos ecológicos del Homo sapiens actual y contrastarlos con ideas, creencias o suposiciones socioculturales. No se pretende adentrarse en los difíciles terrenos de la sociobiología, la ciencia que estudia los comportamientos humanos desde una perspectiva biológica basándose en el hecho de que tales comportamientos tienen un componente genético y están bajo la influencia de la selección natural (Wilson, 2000). La sociobiología requiere de un análisis más profundo, con mayor cantidad de estudios empíricos, por eso no se pretende realizar un recuento histórico ni analizar en detalle los factores que han llevado a que el ser humano actual se presuma separado del resto de organismos. La intención es, más bien, reevaluar nuestro estatus como una especie más del Reino Animal y poner en perspectiva nuestra unión natural con el resto de seres vivos que habitan el planeta Tierra. Para ello, se explicará las principales características ecológicas que definen al Homo sapiens y se analizarán y discutirán las relaciones sociales que se establecen en la especie bajo la luz de la ecología.

\section{Distribución y abundancia}

Toda especie habita en una región o regiones con características ambientales particulares que son tolerables para la especie. De esta forma, el pez payaso, Amphiprion ocellaris, personaje principal de la película Buscando a Nemo, se distribuye naturalmente en arrecifes de coral al sureste de Asia y norte de Australia donde sobrevive en temperaturas cálidas, aguas poco profundas y en medio de los tentáculos con veneno de las anémonas (Froese y Pauly, 2016). Es, en los sitios donde las condiciones son especialmente favorables que una especie alcanza el mayor número de individuos, ya que dedica mayor energía en su reproducción y no sólo en supervivencia y crecimiento corporal. La cantidad de individuos de una especie que se encuentran en un sitio se refiere a la abundancia, la cual es alta en lugares donde la reproducción se maximiza (Begon, Townsend, y Harper, 2006).

Para Homo sapiens, sin embargo, el clima, el relieve, la disponibilidad de recursos y la presencia de otras especies no representan factores limitantes de su distribución o abundancia. Debido a su habilidad de utilizar aditamentos para contrarrestar las condiciones climáticas, construir refugios y emplear herramientas, el humano tiene actualmente una distribución cosmopolita y ha alcanzado, prácticamente en todo sitio en el que habita, altas abundancias (Handel, 2016). Esto implica una presión sobre la biosfera, incluyendo la supervivencia de otros organismos y las relaciones y procesos que se establecen entre estos y el medio circundante. Sin las habilidades mencionadas, 
Homo sapiens estaría limitado a climas tropicales y el número de individuos sería mucho más bajo. Fuera de esta distribución natural el ser humano se comporta, por lo tanto, como una especie invasora, es decir, un organismo que se expande más allá de su ámbito de distribución natural y que sobrevive en una gran variedad de ecosistemas alcanzando altas abundancias (Moutou y Pastoret, 2010).

La percepción que tenemos sobre las especies invasoras es que se debe evitar su expansión descontrolada, tanto en número de individuos como en espacio, ya que sus impactos ecológicos suelen ser negativos (Moutou y Pastoret, 2010). De esta manera, el mismo ser humano también debería regular su propio expansionismo, el cual se produce a costa de la degradación o destrucción de ecosistemas naturales. Resulta necesario, pues, respetar los límites de áreas silvestres no alteradas y fomentar la creación de centros urbanos que incorporen cada vez más elementos naturales.

\section{III. ¿Población o poblaciones?}

Un concepto básico en ecología es el de población, el cual se refiere al conjunto de individuos de la misma especie que se encuentran restringidos geográficamente y que tienen mayor contacto con individuos de este conjunto que con los de áreas distintas (Begon et al., 2006). Aunque parece un concepto sencillo, en la práctica resulta difícil establecer los límites de una población. Un grupo de mariposas azules conocidas como Morpho peleides puede encontrarse restringido en un parche de bosque específico, con lo cual los límites de esta población pueden establecerse fácilmente. Sin embargo, si el bosque es un continuo que se extiende sobre una área geográfica amplia resulta más complicado la delimitación no ambigua de la población, ya que esta puede extenderse por gran parte del terreno y el establecimiento de límites de una o más poblaciones puede volverse subjetivo (Begon et al., 2006).

El mismo problema surge al hablar de una sola o muchas poblaciones de la especie humana desde una perspectiva ecológica. Si bien hemos establecido límites arbitrarios en el planeta con el fin de distinguir países, estos no representan un impedimento ecológico para el contacto de los individuos, aunque sí existen barreras físicas que limitan el libre flujo de individuos de una zona a otra. Por ejemplo, viajar de América hacia otros continentes o incluso dentro de nuestro mismo continente no siempre es factible, a pesar de los modernos medios de transporte con los que contamos actualmente. Aún así, me parece más apropiado considerar una sola población humana que habita en todo el planeta por las siguientes razones:

- Si bien no todos los individuos de una zona pueden movilizarse hacia otros sitios, muchos sí lo hacen aunque sea por periodos cortos. Esto permite el contacto entre individuos incluso de zonas muy separadas geográficamente.

- La cópula entre individuos de zonas separadas ocurre con frecuencia, por lo que hay un constante intercambio genético ( $\mathrm{Li}$ et al., 2008).

- La comunicación entre individuos que se encuentran en zonas separadas puede darse de forma virtual, por lo que existe un contacto social permanente.

\section{Esperanza de vida y reproducción}

La esperanza de vida se define como el número de años que le restan por vivir a un determinado organismo de cierta edad. Usualmente se calcula como un promedio de años al momento del nacimiento de los individuos de una especie. Aunque suele estar relacionado con la longevidad, este último término puede indicar también el número máximo de años que cualquier individuo de una especie puede sobrevivir. Por ejemplo, se conoce que los cuilos o conejillos de Indias (Cavia porcellus) tienen una esperanza de vida al nacer de unos 5 años. No obstante, en cautividad pueden vivir en promedio 8 años y se han registrado individuos de una longevidad máxima de 14 años (Shomer, Holcombe y Harkness, 2015). 
Durante su periodo de vida, todo organismo tiene como fin biológico primordial la transmisión de sus genes a la siguiente generación de individuos (Begon et al., 2006), es decir, lograr reproducirse y dejar descendientes. Cuanto mayor sea la cantidad de descendientes que sobreviven de un individuo, mayor es su éxito reproductivo, sin embargo, debido a limitaciones fisiológicas, anatómicas y de comportamiento existe gran variación en el éxito reproductivo de los individuos de una especie. Retomando el ejemplo de los cuilos, las hembras de esta especie tienen en promedio 3 crías por parto, aunque el rango va desde 1 hasta 8 crías, y pueden tener 3-4 partos por año (Shomer et al., 2015). Para muchas especies este periodo reproductivo es limitado, ya que las hembras tienen un número restringido de óvulos y los machos viejos no pueden competir con los más jóvenes por el acceso a parejas.

La esperanza de vida del ser humano al nacer ha variado a través de la historia, desde 26 años durante la Edad del Hierro (1200 a.C. a 700 d.C.) hasta 67 años en promedio en el año 2010, y varía actualmente según el país, desde 49 años en Suazilandia hasta 83 años en Japón (Galor y Moav, 2007). Asimismo, el récord del ser humano más longevo se ha registrado en 122 años. De esta forma, resulta evidente que la esperanza de vida del ser humano va en crecimiento, aunque depende de las condiciones alimenticias y de salud de los individuos en cada época y lugar. Este incremento está íntimamente relacionado con la Revolución Industrial y los avances médicos, eventos que permitieron la producción masiva de alimentos, medicinas y técnicas reproductivas (por ejemplo, la fertilización in vitro) (Turner et al., 1990). De esta manera, se ha reducido significativamente la mortalidad temprana (infantil), se ha incrementado la supervivencia de individuos enfermos que deberían fallecer naturalmente y se ha otorgado iguales oportunidades reproductivas a una gran mayoría de individuos, incluyendo aquellos con discapacidades físicas.

La especie humana, por tanto, no sigue patrones naturales de comportamiento en aspectos ecológicos como esperanza de vida y reproducción (Corral-Verdugo et al., 2006). Aunque la esperanza de vida al nacer parece haber llegado a un tope y no seguirá aumentando (Dong, Milholland y Vijg, 2016), la prolongada longevidad actual podría generar una población de Homo sapiens con gran cantidad de individuos postrepreductivos (viejos) que no sobrevivirían naturalmente, por lo tanto, no solamente aumenta la cantidad de individuos de la especie en el planeta (abundancia), sino también el tiempo que cada individuo sobrevive, lo cual genera impactos usualmente negativos sobre los ecosistemas naturales.

\section{Competencia y otras interacciones}

La competencia es una de las interacciones ecológicas más comunes entre especies, puede ocurrir tanto entre individuos de la misma especie (competencia intraespecífica) como entre individuos de especies distintas (competencia interespecífica). La competencia ocurre cuando dos individuos buscan obtener un recurso, como alimento, refugio o parejas, que se encuentra de forma limitada en el ambiente, tal que no satisface por igual las necesidades de los individuos que lo requieren (Begon et al., 2006). Un ejemplo es el de los machos de los gorilas occidentales (Gorilla gorilla), los cuales son expulsados de su grupo familiar al alcanzar la juventud y al ser individuos sexualmente maduros, deben competir con otros machos por el acceso a hembras sexualmente reproductivas (Robbins et al., 2004). La competencia es solamente una de muchos tipos de interacciones que ocurren en la naturaleza, entre las cuales se puede mencionar el comportamiento agresivo, la cooperación y el altruismo (esto se refiere al comportamiento de algunos individuos que sacrifican su propia supervivencia u oportunidades reproductivas en beneficio de otros individuos de la misma especie).

La competencia es considerada, con cierta frecuencia, por individuos de la especie humana como una interacción positiva, ya que promueve el mejoramiento de las capacidades de un individuo al enfrentarse a rivales y selecciona a los individuos con las mejores características. Sin embargo, ecológicamente hablando, la competencia es una interacción negativa para los individuos participantes, ya que si el recurso por el cual se pelea no fuese limitante los individuos 
no tendrían la necesidad de competir y podrían utilizar la energía usada en competencia para reproducción o crecimiento corporal. Aún así, la competencia es posiblemente la interacción más frecuente en la población humana, tanto a nivel individual como a nivel colectivo en las sociedades. Usualmente, la competencia se combina con comportamientos como la agresividad, generando efectos nocivos como estrés, ansiedad y miedo, para los seres involucrados (Kohn, 1992). De esta manera, existen otras interacciones que sí son beneficiosas para los individuos que las ejecutan, como la cooperación y el altruismo, aunque suelen ocurrir con menor frecuencia.

\section{Relaciones socioculturales según la ecología de Homo sapiens}

El ser humano es una especie altamente social, motivo por el cual se agrupa en asentamientos que pueden tener grandes cantidades de individuos conviviendo juntos en áreas geográficas relativamente pequeñas, es decir, concentrados en altas densidades. Tales centros urbanos se han multiplicado rápidamente en los últimos 200 años (Cohen, 2006), llegando a establecerse incluso en sitios remotos como Alaska. Estos centros urbanos suelen ser sitios desprovistos de elementos bióticos nativos, por lo que el contacto con la naturaleza es reducido. De esta forma, los seres humanos que viven en grandes centros urbanos son más propensos a desligarse del entorno natural, lo cual genera actitudes de desinterés, miedo o incluso repudio hacia otras criaturas.

La aglomeración de personas en los centros urbanos incrementa e intensifica las interacciones entre individuos, por lo que salen a relucir comportamientos que pueden pasar inadvertidos por su baja ocurrencia en lugares con densidades más bajas. Resultan impactantes y chocantes los comportamientos agresivos, los cuales producen desequilibrio tanto a nivel individual (una persona puede tener repercusiones físicas o psicológicas provocadas por una agresión) como a nivel social (se debe invertir gran cantidad de recursos y energía para controlar este tipo de comportamientos). Si en centros urbanos pueden aparecer comportamientos dañinos durante relaciones humano-humano, no es de extrañar que surjan individuos con baja capacidad para relacionarse adecuadamente con otras especies.

La tendencia al crecimiento que mantiene la población humana indica que podría aumentar la creación de asentamientos, la densidad de los centros urbanos y la frecuencia de comportamientos nocivos (Nentwig, 1999). Si bien los comportamientos beneficiosos también podrían incrementarse, los efectos deletéreos de los comportamientos dañinos opacan en gran medida los efectos positivos de, por ejemplo, la cooperación y el altruismo. Asimismo, la competencia entre individuos de una sociedad y entre sociedades también impacta negativamente, ya que se busca acaparar recursos limitados utilizando medios o estrategias sin considerar los efectos sobre los rivales. Es así como actos cotidianos que involucran unos pocos individuos (por ejemplo, manejar en carretera compitiendo con otros choferes) hasta acontecimientos a escala regional (por ejemplo, guerras por territorio) tienen consecuencias variadas, desde altos niveles de estrés hasta un aumento de la tasa de mortalidad. Ante el incremento que se espera de la población en un futuro y el recrudecimiento de las condiciones climáticas, se podría predecir que la competencia y sus efectos negativos se intensificarán.

El problema se agrava al considerar que más individuos sobreviven hasta edades avanzadas que, de forma natural, posiblemente no ocurriría. Esto provoca que se retengan y se transmitan (tanto a nivel infeccioso como genético) enfermedades que de otro modo podrían desaparecer o disminuir su prevalencia (Jones et al., 2008). Esta retención de enfermedades a nivel poblacional puede deteriorar el $\mathrm{ADN}$ y generar individuos debilitados inmunológicamente. El anhelo humano de alargar su esperanza de vida, en muchas ocasiones sin tomar en cuenta la calidad de vida que se lleva, provoca que se destinen grandes cantidades de recursos y energía en la creación de medicinas y tratamientos. No obstante, los impactos de un mayor número de supervivientes y de una mayor esperanza de vida pueden resultar alarmantes, tanto ambiental como socialmente. 
Una mayor abundancia y densidad de humanos en casi todos los rincones del planeta, además de las interacciones sociales mencionadas anteriormente, genera invariablemente un aumento en el uso de los recursos naturales y una mayor contaminación. Esto produce ambientes degradados que reducen la calidad de vida de las personas. Además, las relaciones de los seres humanos con individuos de otras especies también se vuelven inadecuadas, lo cual afecta negativamente la supervivencia de otros organismos y provoca un deterioro general de los ecosistemas.

\section{Conclusiones}

Ecología es el estudio de las interacciones de los organismos entre sí y de estos con su entorno. En este trabajo se analizaron las características ecológicas del ser humano y se resaltó el hecho de que el Homo sapiens se comporta como una especie invasora con interacciones dañinas hacia los ecosistemas y especies con las cuales convive. Toda especie tiene un efecto sobre los elementos abióticos o sin vida (por ejemplo, el suelo, el agua, el aire, etc.) y los bióticos o con vida (por ejemplo, animales, plantas, hongos, etc.) que se encuentran en el medio donde se desenvuelve la especie. No obstante, el impacto del ser humano supera significativamente el de cualquier otra especie. Este impacto incluye la extinción de otros organismos, la contaminación de los sistemas abióticos, la degradación, erosión y fragmentación de los hábitats (Steffen et al., 2011).

Debido a los impactos negativos que la expansión del ser humano en el planeta ha tenido sobre el entorno, resulta vital una regulación y ordenamiento territorial a nivel local, para la construcción vertical y el aprovechamiento eficientemente del terreno según el tipo de suelo. Además, es imperativo un control de la tasa de crecimiento poblacional, especialmente en países en vías de desarrollo donde esta tasa suele ser alta. Tales cambios y regulaciones no son fáciles de llevar a la práctica, pero hay precedentes sobre ordenamiento territorial ecológico (Wong-González, 2009) y control de la natalidad (Scharping, 2003) que deben servir de ejemplo. Al estar nuestra especie compuesta por una única población, el esfuerzo para lograr tales regulaciones debe expandirse globalmente, liderado por entidades supranacionales como la Organización de las Naciones Unidas y acompañado por organizaciones sin fines de lucro como la World Wildlife Fund.

$\mathrm{Al}$ igual que cualquier otra especie del planeta Tierra, el ser humano tiene características que lo hacen único y particular y le designan una función específica en el ambiente. Tales características incluyen un cerebro proporcionalmente grande que le confiere mayor inteligencia que a otras criaturas, la posición de la laringe y las cuerdas vocales que le permiten establecer complejos sistemas de comunicación, así como un pulgar oponible que le permite tomar y manipular objetos con destreza. El ser humano tiene el potencial, por lo tanto, de utilizar sus facultades para mantener un equilibrio ecológico en el ambiente, realizar acciones que preserven los recursos naturales y a las especies, así como de reducir los impactos negativos sobre los ecosistemas. Sin embargo, el hecho de considerarse como un elemento separado de su entorno y de tener la noción de que todo lo que se encuentra a su alrededor es aprovechable a su conveniencia, provoca que los individuos de nuestra especie muestren desinterés por el bienestar de otros seres vivos y del medio ambiente en general. Se requiere una revaloración de nuestro estatus como especie, la relación con el entorno y los efectos de estas acciones. 


\section{Referencias}

Begon, M., Townsend, C. R., y Harper, J. L. (2006). Ecology: from individuals to ecosystems (4 ed.). Oxford, Reino Unido: Blackwell Publishing.

Cohen, B. (2006). Urbanization in developing countries: current trends, future projections, and key challenges for sustainability. Technology in Society, 28, 63-80.

Corral-Verdugo, V., Frías, M., Fraijo Sing, B., y Tapia Fonllem, C. (2006). Rasgos de la conducta antisocial como correlatos del actuar anti y proambiental. Medio Ambiente y Comportamiento Humano, 7, 89-103.

Darwin, C. R. (1872). The expression of the emotions in man and animals. Londres, Reino Unido: John Murray.

Dhont, K., Hodson, G., Costello, K., y MacInnis, C. C. (2014). Social dominance orientation connects prejudicial human-human and human-animal relations. Personality and Individual Differences, 61-62, 105-108.

Dong, X., Milholland, B. y Vijg, J. (2016). Evidence for a limit to human lifespan. Nature, 538, 257-259.

Franklin, A., y White, R. (2001). Animals and modernity: changing human-animal relations, 1949-98. Journal of Sociology, 37, 219-238.

Froese, R., y Pauly, D. (Eds.). (2016). FishBase: World Wide Web electronic publication. Recuperado de http://www.fishbase.org.

Galor, O., y Moav, O. (2007). The Neolithic revolution and contemporary variations in life expectancy. Rhode Island, Estados Unidos: Brown University, Working Paper.

Handel, S. N. (2016). The ecology of human beings (Homo sapiens) and habitat design. Ecological Restoration, 34, 83-86.
Jones, K. E., Patel, N. G., Levy, M. A., Storeygard, A., Balk, D., Gittleman, J. L., y Daszak, P. (2008). Global trends in emerging infectious diseases. Nature, 451, 990-993.

Li, J. Z., Absher, D. M., Tang, H., Southwick, A. M., Casto, A. M., Ramachandran, S., Cann, H. M., Barsh, G. S., Feldman, M., Cavalli-Sforza, L. L., y Myers, R. M. (2008). Worldwide human relationships inferred from genome-wide patterns of variation. Science, 319, 1100-1104.

Kohn, A. (1992). No contest: the case against competition (2 ed.). Nueva York, Estados Unidos: Houghton Mifflin.

Moutou, F., y Pastoret, P. P. (2010). Defining an invasive species. Revue Scientifique et Technique (International Office of Epizootics), 29, 37-45.

Nentwig, W. (1999). The importance of human ecology at the threshold of the next millennium: how can population growth be stopped? Naturwissenschaften, 86, 411-421.

Robbins, M. M., Bermejo, M., Cipolletta, C., Magliocca, F., Parnell, R. J., y Stoke, E. (2004). Social structure and life-history patterns in Western Gorillas (Gorilla gorilla gorilla). American Journal of Primatology, 64, 145-159.

Scharping, T. (2003). Birth control in China 19492000: Population policy and demographic development. Nueva York, Estados Unidos: RoutledgeCurzon.

Shomer, N. H., Holcombe, H., y Harkness, J. E. (2015). Chapter 6 - Biology and diseases of guinea pigs. En Fox, J. G., Anderson, L. C., Otto, G. M., Pritchett-Corning, K. R., y Whary, M. T. (Eds.), Laboratory animal medicine (3 ed.) (pp. 247-283). Cambridge, Estados Unidos: Academic Press. 
Steffen, W., Persson, A., Deutsch, L., Zalasiewicz,

J., Williams, M., Richardson, K.,...Svedin, U. (2011). The Anthropocene: from global change to planetary stewardship. AMBIO, 40, 739-761.

Turner, B. L. II, Clark, W. C., Kates, R. W., Richards, J. F., Mathews, J. T., y Meyer, W. B. (Eds.). (1990). The Earth as transformed by human action: Global and regional changes in the biosphere over the past 300 years. Nueva York, Estados Unidos: Cambridge University Press.

Whiten, A. (2011). The scope of culture in chimpanzees, humans and ancestral apes. Philosophical Transactions of the Royal Society B, 366, 997-1007

Wilson, E. O. (2000). Sociobiology: the new synthesis, 25th anniversary edition. Massachusetts, Estados Unidos: Belknap Press.

Wimmer, R., Kirsch, S., Rappold, G. A., y Schempp, W. (2002). Direct evidence for the Homo-Pan clade. Chromosome Research, 10, 55-61.

Wong-González, P. (2009). Ordenamiento ecológico y ordenamiento territorial: retos para la gestión del desarrollo regional sustentable en el siglo XXI. Estudios Sociales, 17, 9-39. 